\title{
Lidil
}

Revue de linguistique et de didactique des langues

$44 \mid 2011$

Langues, minor(is)ations et marginalisations

\section{Insécurité linguistique et réseaux sociaux denses ou isolants : le cas de femmes maghrébines dans la tourmente}

\section{Luc Biichlé}

\section{(2) OpenEdition Journals \\ Édition électronique \\ URL : https://journals.openedition.org/lidil/3133 \\ DOI : 10.4000/lidil.3133 \\ ISSN : 1960-6052 \\ Éditeur \\ UGA Éditions/Université Grenoble Alpes}

Édition imprimée

Date de publication : 15 décembre 2011

Pagination : 13-26

ISBN : 978-2-84310-212-7

ISSN : $1146-6480$

Référence électronique

Luc Biichlé, «Insécurité linguistique et réseaux sociaux denses ou isolants : le cas de femmes

maghrébines dans la tourmente », Lidil [En ligne], 44 | 2011, mis en ligne le 15 juin 2013, consulté le 21 septembre 2021. URL : http://journals.openedition.org/lidil/3133; DOl : https://doi.org/10.4000/lidil. 3133 


\title{
Insécurité linguistique et réseaux sociaux denses ou isolants : le cas de femmes maghrébines dans la tourmente
}

\author{
Luc Biichlé*
}

\begin{abstract}
RÉSUMÉ
Chez certaines femmes migrantes, les structures, denses ou isolantes, des réseaux sociaux semblent aller de pair avec plusieurs formes d'insécurité linguistique. Cet article essaie donc de montrer qu'il existe des liens importants entre le capital social des personnes et leurs représentations et pratiques langagières.
\end{abstract}

\section{ABSTRACT}

For some migrant women, the structures of social networks, dense or insulated, appear to be associated with several forms of linguistic insecurity. This article therefore tries to show that there are important links between the social capital of individuals, their social representations and linguistic practices.

Dans le cadre d'une thèse consacrée aux langues et parcours d'intégration d'immigrés maghrébins en France (Biichlé, 2007), je me suis intéressé au rapport qui existe entre apprentissage ou connaissance du français et intégration. J'ai donc effectué 105 entretiens individuels dans des centres de formation ou autres organismes sociaux de la région Rhône-Alpes auprès de migrants originaires du Maghreb (primo-arrivants ${ }^{1}$ ou installés de longue date). Au fil de mes visites, j'ai rencontré certaines femmes très isolées, qu'il m'aurait été extrêmement difficile, voire impossible, de rencontrer dans d'autres circonstances. Ces femmes

* Laboratoire Identité Culturelle, Textes et Théâtralité, Université d'Avignon.

1. Primo-arrivant signifie « en France depuis moins de deux ans » mais j'ai étendu le critère à toutes les personnes séjournant en France depuis moins de trois ans. 
partageaient apparemment deux traits communs saillants : un réseau social dense ${ }^{2}$ (Milroy, 1987), voire isolant ${ }^{3}$ (Bortoni-Ricardo, 1985), et une insécurité linguistique parfois très forte. Mon propos sera donc d'essayer de montrer l'articulation entre le capital social des personnes (Merklé, 2004), la structure de leurs réseaux sociaux, et leurs représentations ou attitudes envers la langue.

\section{Petits rappels à propos de l'insécurité linguistique}

\section{L'insécurité linguistique}

C'est à William Labov que l'on attribue généralement la paternité de la notion d'insécurité linguistique. Dès 1973, celui-ci déclarait que les New-yorkais « sont convaincus qu'il existe une langue "correcte", qu'ils s'efforceront d'atteindre dans leurs conversations soignées » (1976 : 201), démontrant ainsi la relation que les locuteurs établissaient entre la langue qu'ils estimaient être correcte et leur usage propre; entre la représentation d'une norme et un comportement vis-à-vis de celle-ci.

Je n'entrerai pas dans le détail de la taxonomie des normes (objective, subjective, évaluative, prescriptive, etc.) mais je retiendrai qu'elle s'impose à tous les membres d'une même « communauté linguistique ${ }^{4}$ " (Bourdieu, 1982, commentant Labov), et ce, tout particulièrement en France puisque l'écrit, étalon de celle-ci, y est très prégnant (Gadet, 2003). De cette prégnance de la norme objective (grammaticale) résulte fatalement une grande contrainte sur la norme subjective (représentationnelle) qui, en dépit de sa variabilité selon les individus, induit des attitudes et des comportements particuliers parmi lesquels l'insécurité linguistique.

\section{L'insécurité linguistique féminine}

Dans Sociolinguistique, William Labov mettait en évidence un symptôme typique de l'insécurité linguistique, l'hypercorrection, distinguant au

2. Un réseau est qualifié de relativement dense si bon nombre de personnes auxquelles la personne est liée sont également liées entre elles (Milroy, 1987 : 50. Traduit par mes soins).

3. La structure du réseau de la personne fait que celle-ci n'a que très peu de contacts avec la langue du pays d'immigration.

4. Locution que j'aurais volontiers remplacée par « speech community » (Biichlé, Abouzaid, 2008). 
passage les pratiques masculines et féminines : «Il est certain que l'hypercorrection est plus forte chez les femmes » $(1976: 210)$. À l'époque, il expliquait ce phénomène par une plus grande responsabilité des femmes dans l'ascension sociale de leurs enfants mais, dès 1990, il commençait à envisager une approche différente de la question en abordant le problème sous un angle orienté davantage vers les pratiques masculines : «Dans une stratification sociolinguistique stable, les hommes utilisent plus fréquemment que les femmes des formes linguistiques non standard » (1998: 31). C'est précisément ce changement dans l'approche du phénomène qui va le mener à mettre en corrélation directe l'insécurité linguistique des femmes et l'ascension sociale (1998: 34).

\section{Migration, plurilinguisme et insécurité}

Lors des premiers travaux de William Labov, les phénomènes liés à l'insécurité linguistique étaient appréhendés uniquement à travers des situations de variation au sein d'un espace anglophone mais on retrouve des manifestations analogues et souvent plus fortes lors du contact entre langues (Labov, 1998; Calvet, 1999); par conséquent, tout particulièrement chez les migrants. En effet, de l'inconfortable situation d'être entre deux systèmes résultent immanquablement des conflits identitaires (Lüdi, 1995) et de l'insécurité identitaire (Billiez et al., 2002). Cette dernière se manifeste, entre autres, par un sentiment d'insécurité linguistique dû au poids des représentations sur la langue idéale et sur le sentiment subséquent d'une maitrise insatisfaisante du français (Billiez, ibid.). On peut d'ailleurs retrouver ces différentes manifestations d'insécurité de manière plus exhaustive dans les typologies de Cécile Canut (1995) et Louis-Jean Calvet (1999) fondées sur trois critères (insécurité formelle, statutaire et identitaire) et les combinaisons potentielles entre ces derniers en fonction du contexte, des langues en présence, des personnes, etc. (Cavalli et Coletta, 2003).

De manière lapidaire, je retiendrai donc que les manifestations les plus courantes de l'insécurité linguistique sont l'hypercorrection (Labov, 1976), l'auto-(d)évaluation (Billiez, ibid.), voire le mutisme dans les cas les plus extrêmes (Gadet, 2003).

Pour ce qui concerne les femmes migrantes interrogées dans cet article (66 sur 105 personnes), je limiterai mon analyse aux deux dernières manifestations (auto-(d)évaluation et mutisme) et ne mentionnerai pas 
l'hypercorrection; soit parce que je ne l'ai pas perçue, soit parce les enquêtées n'ont pas produit d'énoncé hyper-corrigé.

\section{Les indices de réseau et les manifestations d'insécurité linguistique}

Esquisser le réseau social de quelqu'un à partir d'un entretien n'est jamais chose aisée mais lorsqu'une personne déclare demeurer chez elle la plupart du temps, n'avoir ni ami ni connaissance en dehors de sa famille nucléaire, ne pas avoir d'emploi, etc., et que tout cela est confirmé par les acteurs sociaux en contact (assistante sociale, formateur, etc.), on peut raisonnablement parler d'indices sur le capital social et, par conséquent, sur la structure du réseau social. C'est également pour cette raison que les éventuels enfants figurent dans les indices de réseau puisque, très souvent, pour les femmes en question, ceux-ci représentent un véritable trait d'union, voire le seul, avec la société d'immigration; ne serait-ce que par les contacts établis en allant les chercher à l'école (Biichlé, 2007).

Les déclarations qui suivent sont respectivement celles de femmes primo-arrivantes (en France depuis moins de trois ans) puis celles de femmes installées en France depuis plus longtemps.

\section{Les déclarations des primo-arrivantes}

Les enquêtées suivantes sont en France depuis moins de trois ans (primoarrivantes), mariées, sans emploi et n'ont pas été scolarisées, à l'exception de l'enquêtée $\mathrm{n}^{\circ} 61$ qui l'a été faiblement.

\begin{tabular}{|c|c|c|}
\hline & $\begin{array}{l}\text { Marques d'insécurité } \\
\text { linguist. }\end{array}$ & Indices réseau \\
\hline$n^{\circ} 4$ & $\begin{array}{l}\text { «J'essaie pas de parler parce } \\
\text { que j'ai honte [...] je peux pas } \\
\text { parler avec les gens». }\end{array}$ & $\begin{array}{l}\text { «Personne (ne m'aide), jamais } \\
\text { je trouve quelqu'un [...] J'ai pas } \\
\text { d'ami ». } \\
\text { - } 2 \text { enfants. }\end{array}$ \\
\hline$n^{\circ} 61$ & $\begin{array}{l}\text { «Je peux pas parler, j'ai honte, } \\
\text { peut-être je fais des fautes [...] } \\
\text { j'ai peur qu'on se moque de } \\
\text { moi ». }\end{array}$ & $\begin{array}{l}\text { «e connais personne ici». } \\
\text { «avec une collègue tunisienne } \\
i c i » \\
\text { - Pas d'enfant. }\end{array}$ \\
\hline
\end{tabular}




\begin{tabular}{|c|c|c|}
\hline$n^{\circ} 20$ & $\begin{array}{l}\text { «J'ai pas confiance en moi } \\
\text { pour parler avec quelqu'un (le } \\
\text { français)». }\end{array}$ & $\begin{array}{l}\text { «'ai pas de copines françaises } \\
{[\ldots] \text { pas de voisins, de voisines }} \\
\text { pour discuter } . \\
\text { - Pas d'enfant. }\end{array}$ \\
\hline$n^{\circ} 47$ & $\begin{array}{l}\text { «e ne comprends pas des fois, } \\
\text { alors je parle pas beaucoup } \\
{[\ldots] \text { j'aime bien parler français }} \\
\text { mais j'ai peur que les gens } \\
\text { rigolent } »\end{array}$ & $\begin{array}{l}\text { «Des dames arabes } y \\
\text { m'aident. } » \\
\text { - } 1 \text { enfant. }\end{array}$ \\
\hline
\end{tabular}

Tableau 1. - Marques d'insécurité ${ }^{5}$ chez les 4 enquêtées primo-arrivantes ${ }^{6}$

Le lexique utilisé par ces quatre femmes montre de manière assez explicite le sentiment d'insécurité linguistique avec d'une part, une autoévaluation négative marquée par l'usage de vocables forts (honte, peur, pas confiance, peux pas) et d'autre part, la préoccupation du regard ou de la réaction d'autrui (peur, moque, rigolent, honte).

Dans les quatre cas, la restructuration post-migratoire du réseau social n'a apparemment pas eu lieu (J'ai pas d'ami; Je connais personne ici; $J$ 'ai pas de copines françaises). Le capital social semble donc faible avec pour conséquence un réseau social très dense et multiplexe, voire isolant pour l'enquêtée $\mathrm{n}^{\circ} 61$ et certainement isolant pour l'enquêtée $\mathrm{n}^{\circ} 4$.

On remarquera d'ailleurs que « j'ai honte » et « j'ai peur» correspondent à « je connais personne » ou «j'ai pas d'ami » (enquêtée $\mathrm{n}^{\circ} 4$ et 61), ce qui, en d'autres termes, signifierait assez logiquement d'ailleurs, que la forme d'insécurité linguistique la plus sévère, le mutisme, correspond au capital social le plus faible, le réseau isolant.

\section{Les déclarations des ex primo-arrivantes (ex)}

Les femmes qui suivent vivent respectivement en France depuis quatre, quinze et vingt-trois ans. Toutes trois sont sans emploi, mariées, avec des enfants et ont été faiblement scolarisées à l'exception de l'enquêtée nº 19ex qui ne l'a pas été.

5. Les marques d'insécurité linguistique sont les vocables ou lexies soulignées.

6. Dans ce tableau, l'ordre des enquêtées est fonction de la sévérité des marques d'insécurité. 


\begin{tabular}{|c|c|}
\hline $\begin{array}{l}\text { Marques } \\
\text { d'insécurité } \\
\text { linguistique }\end{array}$ & 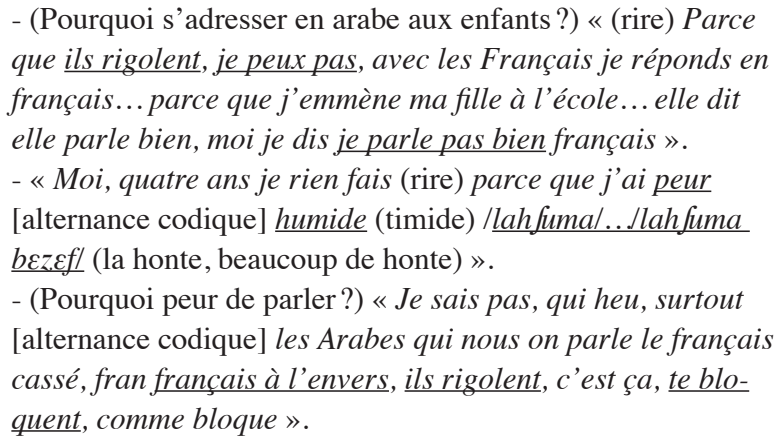 \\
\hline $\begin{array}{l}\text { Indices } \\
\text { réseau }\end{array}$ & $\begin{array}{l}\text { - «Les Français heu, l'hôpital ou le médecin c'est tout, }[\mathrm{X}] \\
\text { c'est tout, ou la voisine et le voisin }[\ldots] \text { parce que je connais } \\
\text { personne français, }[\ldots] \text { toujours avec mon mari } » . \\
\text { (réseau dense, voire isolant) }\end{array}$ \\
\hline
\end{tabular}

Tableau 2. - Marques d'insécurité chez l'enquêtée n³ $32 \mathrm{ex}$

\begin{tabular}{|ll|}
\hline $\begin{array}{l}\text { Marques } \\
\text { d'insécurité }\end{array}$ & - « J'ai peur quand je parle, je crois c'est pas bien $[\ldots]$ je \\
linguistique & pas bien ». \\
& - «Quand je parle avec une Française, je parle bien moi, \\
& mais quand je parle avec un autre Arabe, j'ai peur (qu'il se \\
& moque)». \\
\hline Indices réseaur que quelqu'un comprend & - «Quand les filles (ses filles) parlent avec moi français je \\
& compris je parler». \\
& - (Parler français) «Oui parce que devant l'école il y a des \\
& Français ». \\
& - «Je sors avec mon mari toujours (avant) le magasin et \\
& tout... oui non je sors avec mon mari toujours, je prends le \\
& bus avec mon mari les magasins tout.... \\
& (réseau dense, voire isolant)
\end{tabular}

Tableau 3. - Marques d'insécurité chez l'enquêtée n $33 e x$

$\begin{array}{ll}\text { Marques } & \text { - «Quand je parle à la maison avec mes enfants, y sont } \\ \text { d'insécurité } & \text { tout le temps, y rigolent sur moi, y me dit c'est pas comme } \\ \text { linguistique } & \text { ça, c'est pas comme ça...parce que j'arrive pas à parler } \\ & \text { bien». }\end{array}$




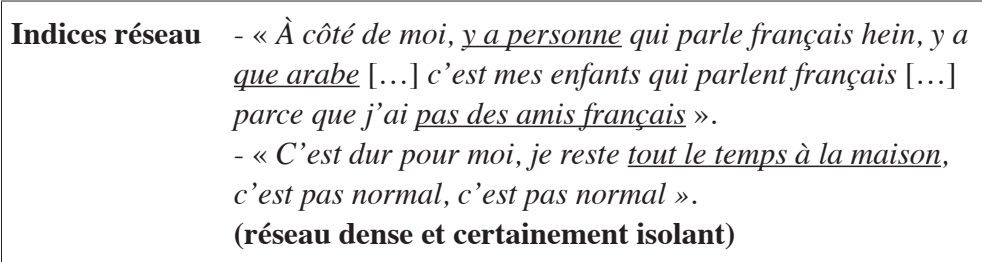

Tableau 4. - Marques d'insécurité chez l'enquêtée nº $19 e x$

À l'instar des enquêtées primo-arrivantes, on retrouve dans ces trois déclarations les marques des auto-évaluations négatives (j'arrive pas, j'ai peur, c'est pas bien, c'est pas juste, je parle pas bien, la honte) et l'appréhension du regard ou de la réaction de l'autre (ils rigolent , y rigolent sur moi, te bloquent, j'ai peur) mais ici, l'autre est identifiable : il y a le regard intra-communautaire (les Arabes, un autre Arabe) et celui des enfants. Le rôle de ces derniers est d'ailleurs souvent à double-tranchant parce qu'ils représentent souvent un nécessaire lien pragmatique entre les parents et la société d'immigration (papiers, traduction, réseau, etc.) mais parfois aussi, comme ici, les détenteurs ou les prescripteurs d'une norme; ce qui conditionne d'ailleurs souvent aussi les modes de communication intrafamiliaux.

Dans les trois cas, la restructuration post-migratoire du réseau social n'a apparemment pas eu lieu non plus en dépit du temps passé en France (l'hôpital ou le médecin c'est tout; je connais personne français; devant l'école il y a des Français; je sors avec mon mari toujours; j'ai pas des amis français; je reste tout le temps à la maison). Le capital social parait donc également faible avec les mêmes conséquences : un réseau social très dense et multiplexe, voire isolant pour les enquêtées 32ex et 33ex, et certainement isolant pour l'enquêtée 19ex.

Enfin, on retrouve l'équation entre «j'arrive pas » et «j'ai peur» qui correspondent à nouveau à «je connais personne » ou «j'ai pas des amis français », le faible capital social et l'insécurité linguistique.

\section{Des causes de non-restructuration du réseau social et des conséquences linguistiques}

\section{Des causes pré-migratoires d'insécurité}

Toutes les femmes de cette enquête sont originaires de zones diglossiques, ce qui renforce souvent le sentiment d'insécurité linguistique (Van den Avenne, 2002) mais de surcroit, la plupart n'a été que faiblement 
ou pas scolarisée en pays d'origine, ce qui peut également prédisposer à l'insécurité, et ce, de manière encore plus prégnante pour les femmes berbères puisqu'elles sont à la base l'enchâssement diglossique ${ }^{7}$ (Calvet, 1987). D'ailleurs, on relèvera que les enquêtées $n^{\circ} 4$ et $n^{\circ} 19 \mathrm{ex}$ sont berbères, respectivement kabyle (Algérie) et tarifit (Maroc), et qu'elles présentent précisément toutes deux une forte insécurité linguistique ainsi que des réseaux apparemment isolants. Il est donc aisé d'imaginer qu'en France, pays où la norme écrite est particulièrement prégnante (Gadet, 2003), ce sentiment soit encore renforcé.

\section{Le rôle des proches dans le contact avec le français}

J'ai évoqué, un peu plus haut, le rôle que se voient parfois octroyer les enfants en tant qu'intermédiaires/traducteurs/évaluateurs entre le(s) parent(s) et la société. Or, pour quelques femmes comme l'enquêtée $\mathrm{n}^{\circ} 15 \mathrm{ex}$, la place de l'enfant-traducteur est si centrale qu'il en résulte une perte significative d'autonomie, un usage quasi inexistant du français et une insécurité linguistique très proche du mutisme :

J'ai besoin pour le la français parce que je vais, je parti à la docteur, je parti à les marchés un peu, je parti les commissions, je parti les tout, alors je toujours emmener ma fille.

Je vais commissions, je vais acheter, j'emmener ma fille [...] et toujours l'interprète pour moi, [...] toujours, je parter le docteur, je parter l'hôpital, je parter tout (avec ma fille) [...] parce que parler pas bien.

On remarquera au passage que les lieux mentionnés donnent un bon aperçu des endroits de contact avec le français, et, par conséquent, précisent la structure du réseau social de la personne.

Pour d'autres femmes, c'est la répartition traditionnelle des rôles dans le couple qui paraît conditionner le rapport à la nouvelle langue puisque certaines enquêtées semblent renoncer à l'usage du français, laissant le mari s'exprimer à leur place :

Enquêtée n 29 : «Oui heu vot'mari, c'est tout (rire) fermer la bouche (rire) ».

Enquêtée $\mathrm{n}^{\circ} 53$ : «Je laisse mon mari qui parle, moi je parle pas ».

7. Louis-Jean Calvet utilise la lexie « diglossie enchâssée » (1987: 47) pour signifier que dans la hiérarchie des variétés, le berbère se situerait en bas de l'échelle, «au-dessous » de la variété basse, de l'arabe dialectal en l'occurrence. 
Enfin, il semble que la faiblesse du capital social de certaines femmes détermine les liens potentiels au point que, en dehors des éventuels enfants, le mari soit quasiment l'unique interlocuteur :

Enquêtée ${ }^{\circ} 32 \mathrm{ex}:$ :Toujours à tout seule avec mes enfants mon mari c'est tout [...] même à la maison mon mari toujours avec moi heu, il dit prends les livres lire heu heu, y a la des mots comprends pas, c'est lui qui spliquer (explique)».

Le rôle des proches est toujours important et, à plus forte raison, lorsque ceux-ci représentent la quasi-totalité du capital social. Toutefois, la contrepartie du service rendu par la traduction systématique ou par l'usage exclusif de la langue d'origine est l'isolement, que ce soit par rapport au français ou à ses locuteurs. La plupart du temps, cet isolement s'accompagne d'une grande insécurité linguistique qui génère, dans les formes les plus aigües, de véritables stratégies d'évitement (Goffman, 1974).

\section{L'emploi, la restructuration du réseau et la langue}

De manière générale, les femmes et les hommes ne sont pas égaux face à l'emploi (Ministère Délégué à la Cohésion Sociale et à la Parité, 2005), mais cette inégalité s'accroît encore dans le cas des femmes migrantes, et tout particulièrement, celui des femmes maghrébines ${ }^{8}$. L'une des conséquences est donc que l'emploi ne participe pas à l'extension ou la restructuration du réseau social, limitant de fait les contacts avec le français.

L'orientation linguistique choisie par le migrant serait d'abord et avant tout tributaire des contacts qu'il établit avec la société d'accueil, bien davantage que de ses compétences linguistiques à l'arrivée. Contrairement à ce que l'on pourrait penser, ce ne serait donc pas tant la compétence

8. «Les Africains sont beaucoup plus mal placés sur le marché de l'emploi, qu'ils soient originaires de pays du Maghreb ou d'Afrique noire. Quels que soient le sexe, l'âge ou l'origine nationale de ces populations, leur taux de chômage ne descend jamais en dessous de 22 \% . Les femmes immigrées sont encore beaucoup plus mal placées que leurs homologues masculins sur le marché de l'emploi. On observe également un léger différentiel au détriment des immigrés originaires d'Algérie. De même, il semble que les femmes originaires d'Afrique noire francophone sont, au-delà de 35 ans, dans une situation plus favorable que celles qui sont originaires du Maghreb » (Rapport d'activité 2001 de la Direction de la population et des migrations, chapitre 1). 
linguistique qui serait décisive en regard du choix linguistique, mais plutôt l'accès à des réseaux francophones et anglophones, parmi lesquels le bureau ou l'usine ne sont pas les moindres. De ce fait, le milieu de travail agit pour les adultes sur le plan linguistique, comme une force intégrative aussi puissante que celle que représente l'école pour l'enfant. (Chamberland, $2005: 179$ )

Les enquêtées ne font pas exception, elles sont sans travail et, à l'inverse des hommes, leur réseau social ne se restructure donc pas grâce à l'emploi. J'ai d'ailleurs eu une autre confirmation de cette articulation entre emploi, réseau et langue lorsque j'ai interrogé Mme Labbé, une conseillère emploi de l'ex ANPE, dont les propos allaient complètement dans ce sens :

Elles ont peur de s'exprimer, de faire des fautes, elles n'osent pas se lancer... [...] En comparaison avec les hommes, les hommes qui ont travaillé, qui ont une expérience professionnelle, ils n'ont pas peur de parler, pas peur de s'exprimer, on voit qu'ils ont été en contact avec le français...

On pourrait donc poser l'articulation entre emploi, réseau et langue comme suit :

\begin{tabular}{|c|c|c|c|}
\hline & Emploi & Restructuration réseau & Insécurité linguistique \\
\hline Hommes & + & + & - \\
\hline Femmes & - & - & + \\
\hline
\end{tabular}

Tableau 5. - Articulation emploi / réseau / langue

\section{Sociabilité, sexe et insécurité}

De manière générale « l'opposition entre "l'extérieur" et "l'intérieur" différencie fortement la sociabilité masculine et féminine » (Mercklé, 2004 : 41). Or, les enquêtées semblent relever de «ménages traditionnels » (Degenne et Forsé, 2004 : 60), ménages dans lesquels la répartition des tâches favorise une sociabilité orientée vers l'extérieur pour les hommes (emploi) alors qu'elle tend à maintenir les femmes dans la sphère du quotidien familial, l'intérieur. Ce mode de fonctionnement, s'il n'est pas l'apanage des Maghrébins, demeure proche de la répartition des tâches et des rôles telle qu'elle est couramment pratiquée dans les couples au Maghreb, à ce bémol près que les réseaux féminins qui existent au Maghreb (famille, voisine, amie, commerces, etc.) disparaissent en France, générant ainsi des formes plus ou moins aigües d'isolement. Les conséquences en terme de structure de réseau sont donc 
souvent les mêmes, densité et multiplexité, avec la plupart du temps un faible usage du français et de l'insécurité linguistique.

Sur le continuum intégrationnel proposé par Luc Biichlé (2009), je situerais donc l'insécurité linguistique comme suit :

$\begin{array}{lll}\begin{array}{l}\text { monolinguisme en } \\ \text { langue d'origine }\end{array} & \text { (bi)plurilinguisme } & \begin{array}{l}\text { monolinguisme } \\ \text { en français }\end{array} \\ \begin{array}{l}\text { ségrégation/marginalisation } \\ \text { (réseaux denses ou isolants) }\end{array} & \begin{array}{l}\text { intégration } \\ \text { (réseaux plus ouverts, moins denses, trous structuraux) }\end{array} \\ \text { INSECURTE linguistique forte } & \text { INSECURITE linguistique moindre ou absente }\end{array}$

Tableau 6. - Schéma de l'insécurité linguistique

\section{Conclusion}

Les situations de ces femmes pourraient paraitre marginales mais elles ne le sont en aucun cas puisqu'au moins $20 \%$ des migrantes interrogées au cours de cette enquête présentaient également des signes plus ou moins forts d'insécurité linguistique. À cet égard, la comparaison entre les primo-arrivantes et les ex-primo-arrivantes montre que, plus que le temps passé en pays d'immigration, c'est la restructuration du réseau social qui confronte à la nouvelle société, ses membres, sa langue, et fait baisser l'insécurité linguistique. En cela, l'inégalité homme/femme en matière d'emploi conjuguée à une sociabilité orientée vers l'intérieur défavorise clairement ces dernières. Alors, même s'il demeure difficile de définir si c'est l'insécurité linguistique qui génère la faiblesse du capital social ou l'inverse, les conséquences sont toujours similaires : réseau social dense et multiplexe, voire isolant, monolinguisme en langue d'origine, autonomie réduite, confrontations identitaires et représentationnelles réduites, et ségrégation ou marginalisation des personnes (Biichlé, 2008a). Toutefois, à l'instar de l'ensemble du processus intégrationnel, le rapport à l'insécurité est dynamique puisque, à partir d'un évènement (changement dans le projet migratoire, emploi, divorce, décès, etc.) les personnes pourront passer d'un réseau dense à un réseau plus favorable (Deprez, 1994), avec, entre autres conséquences, une baisse significative de l'insécurité linguistique (Biichlé, 2008b). Enfin, dans les situations de ces femmes, voire de manière plus générale, plus que le sexe, il me semble que c'est le type de sociabilité qui est source d'insécurité linguistique. 


\section{RÉFERENCES BIBLIOGRAPHIQUES}

BIICHLÉ L. (2007) : Langues et parcours d'intégration de migrants maghrébins en France, thèse de doctorat sous la direction de Jacqueline Billiez, université Stendhal-Grenoble 3.

- (2008a) : «Intégration, langues et réseaux sociaux », dans L. Cadet, J. Goes et J.-M. Mangiante (dir.), Langue et Intégration. Dimensions institutionnelle, socio-professionnelle et universitaire, Peter Lang, collection « GRAMM-R ».

- (2008b) : «La langue et le réseau social », Écarts d'identité, $\mathrm{n}^{\circ}$ 112, p. 94-98.

- (2009) : «Le plurilinguisme c'est l'intégration », Savoir et formation, $\mathrm{n}^{\circ} 73$, p. 32-35.

Birchlé L., Abouzaid M. (2008) : Ainsi meurt la « communauté linguistique », Disponible sur <http://www.u-picardie.fr/LESCLaP/IMG/pdf/Biichle_L._ et_Abouzaid_M._-_Ainsi_meurt_la_communaute_linguistique_pdf $>$

Billiez J., Costa Galligani S., Lucci V., Masperi M., Millet A., Trimaille C. (2002) : «Représentations sociales, pratiques langagières et questions identitaires chez les sujets plurilingues », dans V. Castelloti et D. de Robillard (éds), France, pays de contacts des langues, Cahiers de l'institut de linguistique de Louvain, tome 1, p. 59-78.

BORTONI-RICARDO S. M. (1985) : The urbanization of rural dialect speakers: a sociolinguistic study in Brazil, Cambridge, Cambridge University Press.

Bourdieu P. (1982) : Ce que parler veut dire, l'économie des échanges linguistiques, Éd. Fayard.

Calvet L.-J. (1987) : La guerre des langues et les politiques linguistiques, Payot.

- (1999) : Pour une écologie des langues du monde, Plon.

Cavalli M., Colletta D. (2003) : «Le contexte de la recherche », dans Langue, bilinguisme et représentations sociales au Val d'Aoste, IRRE-VDA (rapport de recherche), Aoste, p. 37-44.

CANuT C. (1995) : Dynamique et imaginaire linguistiques dans les sociétés à tradition orale, thèse de doctorat sous la direction d'Anne-Marie Houdebine, université Paris 3.

Chamberland C. (2005) : «Intégration linguistique des immigrants et intervention en milieu de travail », dans L'intégration des migrants en terre francophone, aspect linguistiques et sociaux (actes du séminaire de Neuchâtel, Suisse, 4-5 décembre 2001), Le Mont-sur-Lausanne, Éditions LEP loisir et pédagogie, p. 173-180.

Degenne A., Forse M. (2004) : Les réseaux sociaux, Paris, Armand Colin [2 $2^{\mathrm{e}}$ édition].

DePrez C. (1994) : Les enfants bilingues : langues et familles, Paris, Didier. GADET F. (2003) : La variation sociale en français, Ophrys. 
GofFMAn, E. (1974) : Les rites de l'interaction, trad. Alain Kihm, Paris, Minuit.

Labov W. (1976) : Sociolinguistique, trad. Alain Kihm, Paris, Minuit.

- (1998) : «Vers une réévaluation de l'insécurité linguistique des femmes, dans P. Singy (dir.), Les femmes et la langue, l'insécurité linguistique en question », Delachaux et Niestlé, p. 25-35.

LÜDI G. (1995) : «L'identité linguistique des migrants en question : perdre, maintenir, changer », dans Changement de langage et langage du changement : aspects linguistiques de la migration interne en Suisse, Lausanne, L'âge d'homme, Métropole 10, p. 205-292.

Merkle P. (2004) : Sociologie des réseaux sociaux, Paris, La Découverte.

Milroy L. (1987) : Language and social network [2 edition], Oxford, Language in Society.

Ministère Délégué À la Cohésion sociale et à la Parité (2005) : 2005 , chiffres clés: l'égalité entre les femmes et les hommes.

VAN DEN Avenne, C. (2002) : «Un récit de ruse, observations à propos de l'insécurité linguistique », dans V. Castelloti et D. de Robillard (éds), France, pays de contacts des langues, Cahiers de l'institut de linguistique de Louvain, tome 1, p. 105-113. 
